\title{
ANÁLISE DE CENÁRIOS PARA IMPLANTAÇÃO DE UMA PEQUENA INDÚSTRIA CERÂMICA NA REGIÃOO AMAZÔNICA
}

Andre Luiz Vilaça do Carmo ' Léo Daiki Shinomiya ' Joner Oliveira Alves '

\section{Resumo}

O trabalho apresenta estudos de cenários técnico e econômico para a implantação de uma pequena fábrica cerâmica, através do estudo de caso de uma prospecção de argila vermelha no interior do Pará. O estudo consistiu na execução de sondagem à trado (ST) na área, para cálculo volumétrico da reserva, e recolhimento de amostra para caracterizações em laboratório. Amostras de argilas foram coletadas e submetidas a análises do teor de umidade, química (fluorescência de raios $\mathrm{X}$ ) e mineralógica (difratometria de raios $\mathrm{X}$ ). Um cenário para uma empresa entre micro e pequena produtora de cerâmica foi estabelecido visando estimativas preliminares para um possível empreendimento. A análise de cenários levou a uma possibilidade de implementação de uma unidade fabril de cerâmica com capacidade produtiva de até 140 mil peças $/ \mathrm{mês}$. Restrições quanto ao tipo de produto desejado e a característica da argila prospectada devem ser consideradas, podendo ser necessário a mistura de argilas de base "gorda", com maior quantidade de argilominerais.

Palavras-chave: Análise de cenários; Indústria cerâmica; Argila vermelha; Pequena empresa.

\section{SCENARIO ANALYSIS FOR THE IMPLANTATION OF A SMALL CERAMIC INDUSTRY IN THE AMAZON REGION}

\begin{abstract}
The work aims technical and economic scenario studies for the implantation of a small ceramic mill, through the case study of a prospecting red clay in the interior of Pará state. The study consisted of an auger drilling on the area for volumetric calculation of the reserve, and sample collection for laboratorial characterization. Clay samples were collected and subjected to analyses of moisture content, chemical composition (X-ray fluorescence) and mineralogical characterization (X-ray diffraction). A scenario of a business among micro and small ceramic mill was established aiming preliminary estimates for a possible enterprise. The scenario analyses led to a possibility of implementing of a ceramic plant with capacity up to 140,000 pieces/month. Constraints among the type of desired product and characteristics of prospected clay should be considered, in which might be necessary a mixture of base clays "fat" with higher amounts of clay minerals.
\end{abstract}

Keywords: Scenario analysis; Ceramic industry; Red clay; Small business.

\section{INTRODUÇÃO}

A argila é um material de ocorrência natural, formada predominantemente por minerais de granulometria fina, que, quando misturada a determinadas proporções de água, torna-se plástica e que endurece ao ser seca ou queimada [I]. Grande parte da argila lavrada no país é destinada às indústrias, tais como, energia, metalomecânica, cerâmica, entre outras. Tal segmento engloba produtos de minerais não metálicos da indústria de transformação, sendo a distribuição setorial do consumo majoritariamente composta por cerâmica vermelha $(33,5 \%)$, construção civil $(21,7 \%)$, pisos e revestimentos $(17,7 \%)$, cimento/artefatos de cimento $(7,4 \%)$, aterro $(2,7 \%)$, cerâmica branca $(2,1 \%)$, construção/manutenção de estradas $(1,5 \%)$, e outros $(13,4 \%)[2,3]$.

'Instituto SENAI de Inovação em Tecnologias Minerais - ISI-TM, Federação das Indústrias do Estado do Pará - FIEPA, Belém, PA, Brasil, E-mail: joner@pq.cnpq.br

2176-1523 (C) 2019 Associação Brasileira de Metalurgia, Materiais e Mineração. Publicado pela ABM. Este é um artigo de acesso aberto distribuído sob os termos da licença Creative Commons CC BY-NC-ND (Attribution-NonCommercial-NoDerivs) - https:// creativecommons.org/licenses/by-nc-nd/4.0\%. 
A indústria de cerâmica vermelha, por ser um setor diretamente ligado a construção civil, se encontra espalhada por todo o território nacional, tanto na forma de pequenas empresas de organização simples até pequenas olarias. São, geralmente, encontradas próximas as suas jazidas de matéria prima e aos mercados consumidores, devido ao seu produto de baixo valor agregado, o que inviabiliza seu transporte a longas distâncias. Esses aglomerados produtivos, são constituídos, tanto pelos núcleos cerâmicos como pelos mercados vizinhos e vem sendo denominados de Arranjos Produtivos Locais (APLs) $[4,5]$.

No estado do Pará, há dois APLs que são amplamente conhecidos. Estão localizados nas cidades de Santarém e São Miguel do Guamá (oeste e nordeste do estado, respectivamente) [4]. Entretanto, há outras localidades de menor expressão, tal como na cidade de Marabá, sudeste do Pará, a qual também apresenta uma considerável produção de cerâmica vermelha [6], bem como regiões em que esta atividade ainda não é notoriamente desenvolvida. Uma dessas regiões é o sul do estado do Pará. Esta região tem como sua principal atividade econômica a agropecuária, seguido de serviços e da indústria. Pensando no desenvolvimento da indústria local, o desenvolvimento das indústrias cerâmicas na região poderia contribuir com as receitas anuais, além da geração de emprego e renda na região [7].

Uma ocorrência de argila na zona rural na região do Araguaia, sul do Pará, despertou o interesse em estudar a possibilidade de uso deste recurso para produção de cerâmica vermelha. $O$ trabalho teve como objetivo a avaliação de cenários técnicos e econômicos para a implantação de uma pequena unidade fabril de cerâmica, considerando esta região como um estudo de caso para prospecção de argila vermelha.

\section{MATERIAIS E MÉTODOS}

\section{I Sondagem da Área}

A execução das sondagens foi realizada em parceria com a GEOTEC Sondagens. A atividade atendeu ao disposto na norma técnica ABNT NBR 9603/86 - Sondagem a Trado e Termo de Referência [8] para a elaboração de diagnósticos, estudo de concepção, projetos básicos e executivos de engenharia, estudos ambientais e exploração de recursos minerais. As investigações diretas foram realizadas de modo a permitir a caracterização dos materiais da subsuperfície e retirada de amostras para caracterização. Amostras da argila foram coletadas na zona rural da cidade de Santana do Araguaia - PA.

\subsection{Caracterização dos Materiais}

A caracterização dos materiais foi realizada em uma amostragem de argila vermelha coletada na zona rural de Santana do Araguaia, estado do Pará. A amostragem foi homogeneizada manualmente e três porções de amostras foram coletadas visando o ensaio em triplicata.

\subsection{Análise de umidade}

Amostras de aproximadamente $5,0 \mathrm{~g}$ das argilas foram dispersas em vidros relógios e levadas a uma estufa com temperatura de $105^{\circ} \mathrm{C}$ por $24 \mathrm{~h}$. Em seguida, as amostras foram pesadas em balança analítica.

\subsubsection{Análise mineralógica}

As amostras foram secadas a $105^{\circ} \mathrm{C}$ por $24 \mathrm{~h}$, cominuídas em moinho de panela Herzog HSM $100 \mathrm{H}$, classificada em peneira manual de 100 mesh e homogeneizada manualmente por pilha cônica. Análises mineralógicas por Difratometria de Raios-X (DRX) foram realizadas em equipamento modelo Empyrean Série 2, tubo de Raios- $X$ cerâmico de Cobre e nível de potência máxima de 2,2 kW, da marca PANalytical. Os dados foram adquiridos através do software Data Collector e a interpretação foi realizada com software HighScore Plus. As condições de análise foram: fenda soller de $0,02 \mathrm{rad}$, filtro $\mathrm{k} \beta$ de $\mathrm{Ni}$, fenda divergente $1 / 4$, fenda antiespalhamento $1 / 2$, spinner I rps, detector $3 \mathrm{~d}$ PIXcel IxI, ângulos de 3,5 a $75^{\circ} 2 \theta$, tamanho do passo 0,02, tempo por passo $55 \mathrm{~s}, 40 \mathrm{kV}$ e $40 \mathrm{~mA}$.

\subsubsection{Análise química}

Amostras foram prensadas com proporção de I para 4 (Aglomerante WAX + Amostra). Análises químicas por Espectroscopia de Fluorescência de Raios-X por Energia Dispersiva (EDXRF) foram realizadas em equipamento modelo Epsilon 3XLE, tubo de Raios-X cerâmico de Ródio e potência máxima de $15 \mathrm{~W}$, da marca PANalytical. Os dados foram adquiridos através do software Epsilon 3 e a interpretação foi realizada com a aplicação Omnian. Também foi realizada perda ao fogo (PF) com I $g$ de amostra em mufla a $1.000^{\circ} \mathrm{C}$ por $\mathrm{Ih}$.

\subsection{Análise de Cenários Técnicos e Econômicos}

A análise de cenários técnicos e econômicos foi baseada em informações de mercado e dados técnicos, não sendo estudadas informações específicas da empresa para geração de um cenário individual. Aspectos considerados nas análises: I) avaliação técnica: estágio de desenvolvimento da tecnologia; competências técnicas; plataforma; complexidade e outros; 2) avaliação econômica: otimista; projetado; pessimista; e 3) construção dos cenários: emprego dos índices VPL - Valor Presente Líquido; TIR - Taxa Interna de Retorno; Payback - Retorno do Investimento. 


\section{RESULTADOS E DISCUSSÃO}

\section{I Estimativa da Capacidade de Extração e Duração da Jazida}

A sondagem a trado ST revelou uma incidência de Argila Vermelha com profundidade média de 0,4 m. A cubagem média do volume estimado para a Argila Vermelha foi de $30.000 \mathrm{~m}^{3}$. A capacidade de extração e a duração da jazida para uma micro e pequena empresa foram estimadas com base no setor cerâmico do norte do estado do Pará [9]. Em uma suposta empresa de porte intermediário (Médio Porte), o consumo e a produção foram estimados através de interpolação dos dados entre uma micro e pequena empresa, como apresentado na Tabela I, a qual apresenta também, a produção de peças.

Considerando o volume estimado de argila vermelha e a produção nominal de uma microempresa (até $100 \mathrm{mil}$ peças/mês), o período de exploração da jazida poderia ser de cerca de 16 anos. Para uma pequena empresa, a qual a produção anual média é de até 300 mil peças/mês, estima-se um período de cerca de 4 anos. Ressalta-se que durante o processo de produção, existem perdas decorrentes do manuseio, tais como, matéria-prima e produto final. Além disso, deve-se considerar o investimento inicial, a demanda de mercado local e os custos de operação envolvidos.

\subsection{Caracterização da Argila}

\subsection{Análise de umidade}

As análises de umidade, realizadas em triplicata, indicaram que os teores de umidade para as três alíquotas foram de I I,40\%, o que infere uma uniformidade das argilas neste aspecto.

\subsubsection{Composição mineralógica}

O difratograma da Argila Vermelha está apresentado na Figura I. A amostra apresentou composição mineralógica composta por: argilomineral caulinita $(7,20$ e 3,52 $\AA$ ) e os minerais gibbsita $(4,84 \AA)$, quartzo $(3,34$ e $4,25 \AA)$ e anatásio $(3,52 \AA)$.

Segundo estudos das propriedades de argilas para uso como cerâmica vermelha [10-12], o limite de plasticidade dos argilominerais aumenta conforme a composição do material apresenta, em ordem crescente, caulinita bem cristalizada, caulinita mal cristalizada, illita e montmorillonita. A amostra de argila vermelha analisada apresenta apenas o argilomineral caulinita, podendo influenciar em uma plasticidade reduzida e, com isto, demandar uma mistura com argilas que apresentem maior volume de argilominerais para emprego industrial.

\subsubsection{Composição química}

O resultado da análise química da amostra é apresentado na Tabela 2. O silício foi o elemento predominante na amostra (55,55\%), sendo a sua presença associada aos minerais quartzo e caulinita. O segundo elemento em maior concentração, o alumínio (32,55\%), possui associação aos minerais caulinita e gibbsita. O Ferro, terceiro elemento mais abundante (2,32\%), é associado ao mineral Hematita (apesar deste não ter sido encontrado), devido à coloração avermelhada da argila. $O$ titânio é associado ao anatásio, e o zircônio não foi associado aos minerais identificados. A Perda ao Fogo (PF), referente a materiais voláteis, como água adsorvida e estrutural, matéria orgânica, carbonatos [13], e apresentou valor de $8,20 \%$.

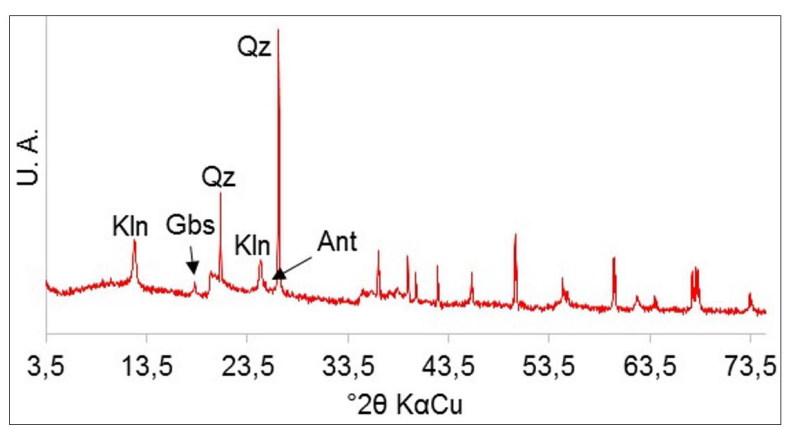

Figura I. Difratograma da argila: Caulinita (Kln), Gibbsita (Gbs), Quartzo (Qz) e anatásio (Ant).

Tabela I. Estimativa de duração da argila conforme o porte de empresa

\begin{tabular}{lccc}
\hline \multicolumn{1}{c}{ Tipo } & $\begin{array}{c}\text { Consumo } \\
\text { (ton/h) }\end{array}$ & $\begin{array}{c}\text { Produção } \\
\text { (mil peças/mês) }\end{array}$ & $\begin{array}{c}\text { Duração } \\
\text { (anos) }\end{array}$ \\
\hline Microempresa & $\mathrm{I}, \mathrm{I}$ a I,4 & $<100$ & $\sim 16$ \\
Médio Porte & $\sim 2,7$ & 80 a 160 & $\sim 8,3$ \\
$\begin{array}{l}\text { Pequena } \\
\text { Empresa }\end{array}$ & 5,5 a 6,5 & 100 a 300 & $\sim 3,7$ \\
\hline
\end{tabular}

Tabela 2. Composição Química da Argila Vermelha

\begin{tabular}{cc}
\hline Elemento & Concentração (\%) \\
\hline $\mathrm{SiO}_{2}$ & 55,55 \\
$\mathrm{TiO}_{2}$ & 1,10 \\
$\mathrm{ZrO}_{2}$ & 0,10 \\
$\mathrm{Al}_{2} \mathbf{O}_{3}$ & 32,55 \\
$\mathrm{Fe}_{2} \mathbf{O}_{3}$ & 2,32 \\
$\mathbf{P F}$ & 8,20 \\
Outros & 0,18 \\
\hline
\end{tabular}

(*): Somatório dos elementos com concentração inferior a 0, I\%. 


\subsection{Análise de Cenários Técnicos e Econômicos}

Atualmente, a região de Santana do Araguaia possui empresas ceramistas. Em regióes próximas, também, podem ser encontradas unidades fabris em Conceição do Araguaia (PA) e Vila Rica (MT).

Considerando que o PIB da região de Santana do Araguaia, em 2013 , foi de aproximadamente $\mathrm{R} \$ 316$ milhões, sendo $43 \%$ pela agropecuária, $34 \%$ por serviços e $23 \%$ pela indústria [14], isto pode representar um potencial mercado para a cerâmica e desenvolvimento de novos empreendimentos da cidade e sua região, como obra de construção de residências, mercados e infraestrutura urbanas e rurais.

Para a implantação de uma unidade fabril de cerâmica, faz-se necessário considerar o investimento inicial com a aquisição dos equipamentos, o transporte destes e dos materiais de construção para galpões, fornos, bem como para a construção da base para a unidade fabril. Uma fábrica de tijolos, basicamente, precisa de área para armazenamento de argila, área de extrusão (sala de máquinas), estufa para secagem dos produtos e fornos para a queima.

Uma análise com base em um negócio intermediário entre uma micro e pequena empresa produtora de cerâmica foi realizada considerando produção estimada de tijolos de 06 (seis) furos $(09 \times 14 \times 19 \mathrm{~cm})$. No local estudado já se tem área suficiente para instalação e posterior ampliação do negócio proposto, além de um volume de argila estimado em pelo menos $30.000 \mathrm{~m}^{3}$, conforme relato da análise de cubagem. A necessidade da adição de uma argila com características diferentes da estudada poderia ser demandada, conforme necessidade do produto.

\subsection{Estimativa de cenários para uma fábrica de tijolo cerâmico}

A proposta de negócio considera a empresa atendendo ao mercado local, considerando que as empresas ceramistas da região estão distantes, deixando espaço para o desenvolvimento desta nova unidade fabril. A empresa, por ser de um porte intermediário, a priori, poderia atender a uma demanda de 140 milheiros de tijolos por mês, com dois fornos do tipo paulistinha, com capacidade de produzir até 20 milheiros de tijolos de 06 furos $(09 \times 14 \times 19 \mathrm{~cm})$, em cada fornada, em um ciclo de 4 dias. Estimativas baseadas em valores de mercado apontam que o milheiro deste produto é comercializado com valores entre $\mathrm{R} \$ 230,00$ e $\mathrm{R} \$ 430,00$ [9].

Considerando a tabela de depreciação de bens da receita federal brasileira, foi estabelecida uma taxa média de $8,0 \%$ a.a. para depreciação dos equipamentos, gal pões, fornos e instalações. $O$ encargo social considerado foi para uma pequena empresa optante pelo SIMPLES NACIONAL [15] e um reajuste salarial anual de aproximadamente $8,3 \%$, baseado na média inflacionária dos últimos 4 anos, contando inicialmente com 6 operários e gerenciado sem custos pelo proprietário da empresa.

Considerando a retração volumétrica após a queima, de 6 a $10 \%$, e perdas que ocorrem durante o processamento $[16,17]$, para cada $\mathrm{m}^{3}$ de argila podem ser fabricados cerca de 874 tijolos. O valor considerado para o milheiro foi de $\mathrm{R} \$ 250,00$, próximo ao mínimo praticado e assim dando margem de aumento no valor do produto. Neste sentido, as estimativas levam em consideração a produção final com um lucro bruto mensal de pelo menos $R \$ 35.000,00$.

O investimento inicial, calculados com base em trabalhos [ 18,19$]$, ficam em torno de $R \$ 290.000,00$ para a construção dos galpões em estruturas metálicas e os fornos, e cerca de $R \$ 263.000,00$ para aquisição dos equipamentos.

Para a estimativa dos cenários econômicos, o Valor Presente Líquido (VPL), a Taxa Interna de Retorno (TIR) e o Payback, foi considerado investimento inicial de $\mathrm{R} \$ 553.000,00$, receita bruta de $R \$ 35.000,00$ e principais custos variáveis de 09 a 12\%. Assim, três cenários foram considerados: $\left.\mathrm{I}^{\circ}\right)$ projetado, considerando o milheiro comercializado a $\mathrm{R} \$ 250,00 ; 2^{\circ}$ ) otimista, considerando a venda do milheiro a $\mathrm{R} \$ 275,00 ; \mathrm{e} 3^{\circ}$ ) pessimista, o valor do milheiro a $R \$ 237,50$ e aumento no custo total em 3,0\% a.a.

Para os três cenários foi suposto que haveria o financiamento de $50 \%$ do investimento inicial a uma taxa de aproximadamente II\% a.a. A taxa de desconto atual é uma porcentagem que abate $o$ valor da VPL, a qual considera as taxas de investimento mais seguro (financiamento), a inflação e o capital financiado. Os resultados para o VPL, TIR e para - Payback são mostrados na Tabela 3.

Nota-se que para o cenário projetado os valores de VPL foram positivos e a TIR foi cerca de $5 \%$ superior à taxa de desconto atual. Este cenário mostra que o investimento é recuperado em torno de 47 meses comercializando o milheiro do tijolo ao valor de $\mathrm{R} \$ 250,00$. Para o cenário otimista, o VPL mostrou-se positivo e a TIR foi cerca de $31 \%$, ou seja, $15 \%$ superior à taxa de desconto atual e o

Tabela 3. Estimativas para os Cenários Econômicos

\begin{tabular}{cccc}
\hline Indicador & Cenário Projetado & Cenário Otimista & Cenário (Pessimista) \\
\hline $\begin{array}{c}\text { Valor do milheiro } \\
\text { comercializado }\end{array}$ & $\mathbf{R} \mathbf{2 5 0 , 0 0}$ & $\mathbf{R} \mathbf{2 7 5 , 0 0}$ & $\mathbf{R} \mathbf{2 3 7 , 5 0}$ \\
Investimento inicial (R\$) & $553.000,00$ & $553.000,00$ & $553.000,00$ \\
Taxa de desconto atual (\%) & 16 & 16 & 16 \\
VPL (R\$) & $55.787,04$ & $191.913,94$ & $-26.359,27$ \\
TIR (\%) & 21 & 31 & 14 \\
Payback (meses) & 47 & 40 & 54 \\
\hline
\end{tabular}


investimento teria retorno em 40 meses. Considerando o cenário pessimista, com custos aumentando em 3\% a.a. e milheiro comercializado a $\mathrm{R} \$ 237,50$, o projeto apresenta uma baixa perspectiva de retorno.

\section{CONCLUSÃO}

A sondagem, com base nos parâmetros estabelecidos, revelou uma cubagem de reserva mineral de aproximadamente $30.000 \mathrm{~m}^{3}$ de argila vermelha. Considerando estimativas baseadas em mercado e literatura, a análise de cenários levou a uma possibilidade de implementação de uma unidade fabril de cerâmica com capacidade produtiva de até 140 mil peças/mês, comercializada no valor mínimo do milheiro de $\mathrm{R} \$ 250,00$. Ressalta-se restrições quanto ao tipo de produto desejado e a característica da argila prospectada, podendo ser necessário a mistura de argilas com maior quantidade de argilominerais.

\section{Agradecimentos}

Os autores agradecem o apoio financeiro do Serviço Brasileiro de Apoio às Micro e Pequenas Empresas (SEBRAE) e fomento de bolsa do Conselho Nacional de Desenvolvimento Científico e Tecnológico (CNPq).

\section{REFERÊNCIAS}

I Guggenheim S, Hillier S, Jaynes W, Maurice P, Pozo M, Velbel M. The Clay Minerals Society Glossary for Clay Science Project. Virgínia: The Clay Minerals Society; 2015 [acesso em 16 mar. 2016]. Disponível em: http://www. clays.org/Clay_Glossary.htm.

2 Brasil. Departamento Nacional de Produção Mineral. Anuário Mineral Brasileiro. Brasília: DNPM; 2010 [acesso em 28 mar. 2016]. Disponível em: http://www.dnpm.gov.br/assuntos/ao-publico/anuario-mineral/arquivos/ANUARIO_ MINERAL_20I0.pdf.

3 Bustamante GM, Bressiani JC. A Indústria Cerâmica Brasileira. Cerâmica Industrial. 2000;5(3):3 I-36.

4 Junior MC, Tanno LC, Sintoni A, Motta JFM, Coelho JM. A Indústria de Cerâmica Vermelha e o Suprimento Mineral no Brasil: Desafios para o Aprimoramento da Competitividade. Cerâmica Industrial. 20I2; I7(I):36-42.

5 Alves JO, Junca E, Espinosa DCR, Tenório JAS. Resíduo do corte de granito: inovação tecnológica para a destinação final. Tecnologica em Metalurgia, Materiais e Mineração. 2015;12:123-128.

6 Santos CVP, Silva AR, Filho MASG, Neto EF, Rabelo AA. Índice de plasticidade e análise racional de Argilas de Marabá (PA) para avaliação das zonas de extrusão. Cerâmica Industrial. 2012;17(2):25-27.

7 Instituto Brasileiro de Geografia e Estatística - IBGE. Rio de Janeiro: IBGE; 2016 [acesso em 20 jan. 2016 ]. Disponível em: https://cidades.ibge.gov.br/brasil/pa/santana-do-araguaia/panorama.

8 Associação Brasileira de Normas Técnicas - ABNT. NBR 9603: Sondagem a Trado. Rio de Janeiro: ABNT; 1986. 6 p.

9 Instituto Nacional de Tecnologia - INT. Avaliação do Setor de Ceramica da Regiao Norte do Estado do Pará Regioes de Abaetetuba, Igarape-Miri e São Miguel do Guama. Rio de Janeiro: EELA; 20I I. (Programa de Eficiencia Energética en Ladrilleras Artesanales de America Latina para Mitigar el Cambio Climaticos). 12 p.

10 Macedo RS, Menezes RR, Neves GA, Ferreira HC. Estudo de argilas usadas em cerâmica vermelha. Cerâmica. 2008;54:4II-4I7.

I I Junior LAG, Varajão AFDC, Souza MHO, Moreno MMT. Estudo Comparativo das Olarias dos Municípios de Alfenas e Areado, MG. Cerâmica. 2012;58(347):404-4II.

12 Grim RE. Clay Mineralogy. New York: McGraw-Hill Book; 1968.

13 Santos PS. Tecnologias de Argilas. São Paulo: Edgard Blücher; 1975.

14 Costa E, Pires G. Diagnóstico Socioeconômico e Ambiental da Região de Integração do Araguaia. Pará: FAPESPA; 2016 [acesso em 16 abr. 2016]. Disponível em: http://www.seplan.pa.gov.br/sites/default/files/PDF/ppa/ppa20I62019/perfil_regiao_araguaia.pdf.

15 Brasil. Ministério da Fazenda. Simples - Microempresa (ME) e Empresa de Pequeno Porte (EPP). Brasília: Ministério da Fazenda; 2005 [acesso em 20 mar. 2016]. Disponível em: http://www8.receita.fazenda.gov.br/simplesnacional.

16 Batista VR, Nascimento JJS, Lima AGB. Secagem e queima de tijolos cerâmicos maciços e vazados incluindo variações dimensionais e danos estruturais. Revista Eletrônica de Materiais e Processos. 2008;3(I):46-6I.

17 Shinomiya LD, Gomes JO, Alves JO. Planejamento de cenários para uso de resíduos industriais: aplicação para lama vermelha. Revista Gestão em Engenharia. 2015;2:43-66. 
I8 Silva AC. Análise de Viabilidade Econômica Financeira para a Implantação de uma Central de Massa em uma Indústria Cerâmica de Itaboraí, RJ [dissertação]. Rio de Janeiro: Centro Federal de Educação Tecnológica Celso Suckow da Fonseca; 2012.

19 Serviço Brasileiro de Apoio às Micro e Pequenas Empresas - SEBRAE. Fornos do Setor de Cerâmica Vermelha do Rio Grande do Norte. Natal: SEBRAE; 20I4. (Relatório de Assessoria Técnica e Tecnológica). 20 p.

Recebido em: 6 Mar. 2018

Aceito em: II Jun. 2018 